\title{
Differential Stability of Aurein 1.2 Pores in Model Membranes of Two Probiotic Strains
}

\author{
Galo E. Balatti, Carmen Domene, M. Florencia Martini, and Monica Pickholz*
}

Cite This: J. Chem. Inf. Model. 2020, 60, 5142-5152

Read Online

ABSTRACT: Aurein 1.2 is an antimicrobial peptide from the skin secretion of an Australian frog. In the previous experimental work, we reported a differential action of aurein 1.2 on two probiotic strains Lactobacillus delbrueckii subsp. bulgaricus (CIDCA 331) and Lactobacillus delbrueckii subsp. lactis (CIDCA 133). The differences found were attributed to the bilayer compositions. Cell cultures and CIDCA 331-derived liposomes showed higher susceptibility than the ones derived from the CIDCA 133 strain, leading to content leakage and structural disruption. Here, we used molecular dynamics simulations to explore these systems at the atomistic level. We hypothesize that if the antimicrobial peptides organized
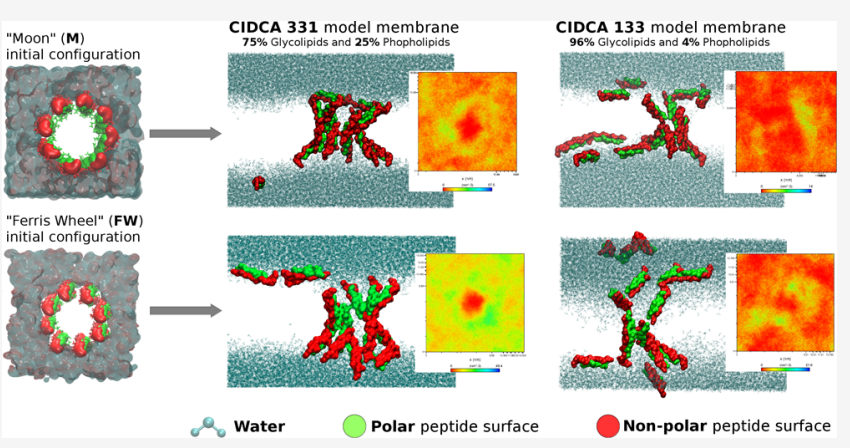
themselves to form a pore, it will be more stable in membranes that emulate the CIDCA 331 strain than in those of the CIDCA 133 strain. To test this hypothesis, we simulated preassembled aurein 1.2 pores embedded into bilayer models that emulate the two probiotic strains. It was found that the general behavior of the systems depends on the composition of the membrane rather than the preassemble system characteristics. Overall, it was observed that aurein 1.2 pores are more stable in the CIDCA 331 model membranes. This fact coincides with the high susceptibility of this strain against antimicrobial peptide. In contrast, in the case of the CIDCA 133 model membranes, peptides migrate to the water-lipid interphase, the pore shrinks, and the transport of water through the pore is reduced. The tendency of glycolipids to make hydrogen bonds with peptides destabilizes the pore structures. This feature is observed to a lesser extent in CIDCA 331 due to the presence of anionic lipids. Glycolipid transverse diffusion (flip-flop) between monolayers occurs in the pore surface region in all the cases considered. These findings expand our understanding of the antimicrobial peptide resistance properties of probiotic strains.

\section{INTRODUCTION}

The study of factors that affect the viability of probiotic cells is of particular interest in food and health sciences. Probiotics are living bacterial cells that confer health benefits to their host. ${ }^{1}$ In humans, the intestinal tract hosts a plethora of probiotics that exert beneficial effects on our digestive and immune systems. ${ }^{2}$ Lactobacillus delbrueckii subsp. lactis (CIDCA 133), which is able to counteract the action of pathogenic microorganims, ${ }^{3-6}$ protect intestinal mucose, ${ }^{7}$ or stimulate phagocytosis, ${ }^{8}$ is among this type of bacteria. CIDCA 133 in vitro experiments have shown its resistance to cationic extracts of cultured human enterocytes and to antimicrobial peptides (AMPs) such as $\beta$-defensins, present in the gastrointestinal tract.

AMPs are molecules found in virtually all domains of life. They have activity against a wide variety of biological membranes, and they are important components of innate immunity in vertebrate and invertebrate systems. They show remarkable structural and functional diversity with the ability to either kill microbial pathogens directly or act indirectly by modulating the host defense systems. ${ }^{9-11}$ The overall mechanism of action described for most of the AMPs is based on their interaction with plasmatic membranes where they can alter the physical integrity of the bacterial cell membrane at different degrees. ${ }^{12-16}$ However, other ways of action of AMPs are reported in literature many of which affect membrane-associated biosynthesis or the interaction with intracellular targets. ${ }^{17,18}$

Generally, AMPs are short cationic peptides ( $<40$ amino acids), with a net charge ranging from +1 to $+9 .{ }^{12,19}$ They adopt $\alpha$-helix, $\beta$-strand, loop, or extended conformations, and they are classified into four categories according to their secondary structure. ${ }^{12}$ The first group encompasses peptides that in solution show random coil conformations, but they reorganize in $\alpha$-helical structures when they interact with lipid bilayers. Aurein 1.2, with sequence GLFDIIKKIAESF-NH2, belongs to this category, and it is found in the skin of the Australian tree frogs. ${ }^{20}$ It has a net positive charge and an

Received: July 27, 2020

Published: August 20, 2020 
overall hydrophobic moment of $\mu \mathrm{H}=6.77$ in the WimleyWhite interfacial hydrophobicity scale, ${ }^{21}$ calculated with the totalizer module of the Membrane Protein Explorer software. ${ }^{22}$ In its wild-type form, the C-terminus of aurein 1.2 is amidated by post-translational modification, ${ }^{20}$ which is a feature with relevance to its biological activity as it facilitates the interactions of the peptide with the lipid membrane. ${ }^{23}$ The amphipathic character of aurein 1.2 is documented by circular dichroism (CD) experiments, which have shown that aurein 1.2 adopts either an $\alpha$-helical or random coil conformation depending on whether it is in the membrane or aqueous environment. $^{24}$

The mechanisms of action of aurein 1.2 are not entirely understood, and previous computational and experimental studies described different behaviors of the peptide depending on the concentration and bilayer composition. It has been reported that the peptide can alter the target bilayer superficially, by interacting with lipid headgroups, ${ }^{25}$ pulling out anionic lipids, ${ }^{26}$ inducing reversible curvature in the membrane, ${ }^{27}$ or promoting lateral segregation of anionic lipids, thus, leading to membrane defects. ${ }^{28}$ These surface effects of aurein 1.2 on bilayers could be concomitant with membrane leakage, which hinders the biological function of the membrane and alters the cell viability. ${ }^{28}$ The interaction of the peptide with the membrane surface can also result in a carpet-based mechanism triggered by a certain peptide concentration threshold that depends on the membrane composition. $^{23,29,30}$ Our previous computational studies of the ways of action of aurein 1.2 reported its capability to form pore structures. ${ }^{21,31}$ In this respect, during evolution, prokaryotic cells have developed strategies to counteract antimicrobial activity, and the bacterial response to antibiotic aggression is the prime example of bacterial adaptation and the pinnacle of evolution. One of these strategies relies on the composition of the cell membrane, which dictates the peptide-lipid interaction. $^{32-34}$ It is exhaustively reported that some bacteria reduce their net surface charge, tempering the electrostatic interactions between the peptide and the cell membrane. ${ }^{32-35}$ There are other properties of the bilayer associated with AMP resistance such as the increase of membrane fluidity or the negative curvature induced on the surface. ${ }^{27,36-39}$

Aurein 1.2 interacts with some preference with bilayers composed of anionic over zwitterionic lipids. Specifically, mixtures of POPG/POPC vesicles were more vulnerable to the aurein 1.2 lytic action in comparison with pure POPC vesicles, $^{30}$ and nuclear magnetic resonance (NMR) experiments showed a preferential interaction of aurein 1.2 with vesicles formed by mixtures of DMPC/DMPG in comparison with pure DMPC. ${ }^{24}$ Likewise, negatively charged DMPG bilayers were perturbed to a greater extent by aurein 1.2 than neutral DMPC bilayers as shown by differential scanning calorimetry (DSC) experiments. ${ }^{40}$

Recently, we reported that liposomes derived from the CIDCA 133 strain are more resistant against the action of three anuran AMPs (citropin 1.1, maculatin 1.1, and aurein 1.2) than those derived from the CIDCA 331 strain. $^{41}$ The CIDCA 133 strain showed a greater minimal inhibitory concentration (MIC) and a remarkable cell viability (flow cytometry experiments) compared to the CIDCA 331 strain. In addition, the CIDCA 133 was significantly inhibited by the three peptides in cell culture kinetics studies. ${ }^{41}$ These observations suggest that the degree of resistance of the
CIDCA 133 strain to AMPs is higher compared to the CIDCA 331 strain, both, in the entire cells and CIDCA-derived liposomes. Considering these observations, a plausible explanation for the characteristic AMP resistance of the CIDCA 133 strain may be attributed to the membrane composition and, particularly, to a high carbohydrate content and fluidity as a result of a significant unsaturated lipid content. In this respect, the main differences between the CIDCA 133 and CIDCA 331 strains in terms of composition are the glycolipid/phospholipid ratio $(\mathrm{GL} / \mathrm{PL}=23.33$ for CIDCA 133 and 3.08 for CIDCA 331) and the degree of saturation of the lipid tails ( $\%$ uns of 70.2 and 42.8 , respectively). ${ }^{42-44}$

Computer simulations are a powerful tool to characterize the mechanisms of interaction of AMPs with model membranes, and many studies have been already reported in the literature studies. ${ }^{33,45-48}$ Previously, we used molecular dynamics (MD) simulations at the coarse grain level to explore the interactions of aurein 1.2 with eukaryotic ${ }^{21}$ and prokaryotic ${ }^{31}$ model membranes. These studies were further extended to investigate the nature of transient aurein pores in membranes with different GL/PL ratios. ${ }^{49}$

In the present study, extensive all-atom MD simulations have been performed to assess the stability of preassembled pores of aurein 1.2 in model membranes of the CIDCA 133 and the CIDCA 331 strains to shed light on the differential experimental susceptibility that we observed between strains.

\section{METHODOLOGY}

Model Membranes. The membrane composition of the models was selected based in experimental data available for the CIDCA 331 and the CIDCA 133 strains. ${ }^{42-44}$ A summary is presented in Table 1 . The model membranes were

Table 1. Composition of the Model Membranes Considered in This Study

\begin{tabular}{cccc} 
& \multicolumn{3}{c}{ membrane composition } \\
\cline { 2 - 4 } system & \multicolumn{1}{c}{ phospholipids } \\
\cline { 2 - 4 } C331 & 384 OPMG (75\%) & \multicolumn{1}{c}{ POPG } & \multicolumn{1}{c}{ CL2 } \\
C133 & 490 DOMG (96\%) & $14(3 \%)$ & $32(6.25 \%)$ \\
& & & $4(1 \%)$ \\
\hline
\end{tabular}

composed of 1-palmitoyl-2-oleoyl-sn-glycero-3-phospho-( $1^{\prime}$ rac-glycerol) (POPG) and cardiolipin (CL2) with net charges of -1 and -2 , respectively, and dioleoyl-3-monogalactosyl-snglycerol (DOMG) or 1-palmitoyl-2-oleoyl-3-monogalactosylsn-glycerol (OPMG) in the C133 and C331 models, respectively. Chemical formulas of these molecules are depicted in Figure 1.

Pore Assembly. The aurein 1.2 structure obtained from NMR experiments ${ }^{20}$ (code: 1VM5) was retrieved from the Protein Data Bank. ${ }^{50}$ The ionization states corresponding to neutral $\mathrm{pH}$, the natural form of the peptide, were considered for all the amino acids, with the C-terminal amidated and the $\mathrm{N}$-terminal protonated.

In previous CG simulations, aurein 1.2 formed pore structures composed of 16 peptides on average per pore that had well-defined characteristics such as the disposition of molecules per leaflet with a preferential $35^{\circ}$ tilt angle with respect to the membrane normal in agreement with NMR experimental observations. ${ }^{51}$ It was observed that the amidated termini were oriented toward the hydrophobic core of the 

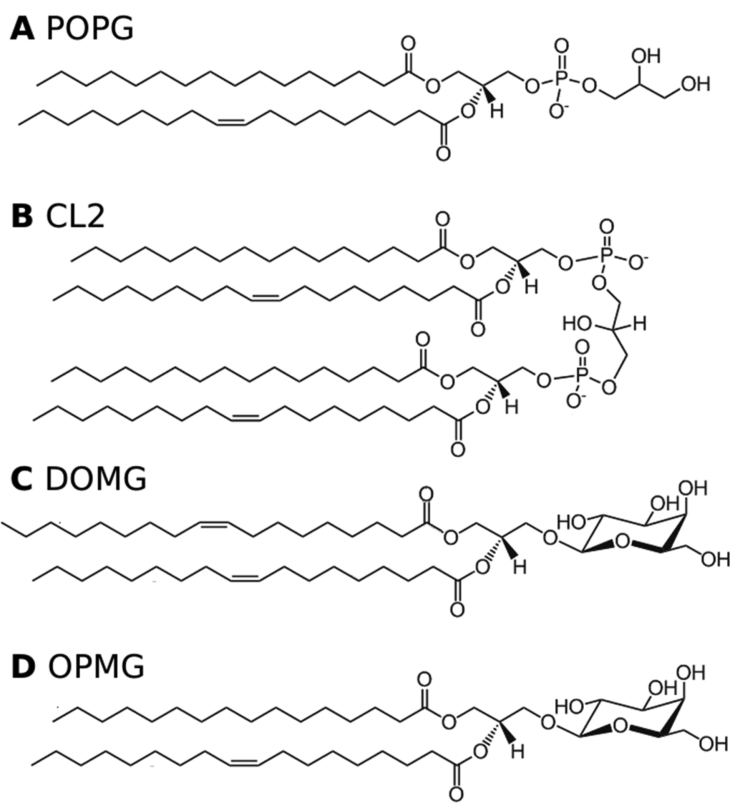

Figure 1. (A-D) Chemical structures of the lipids considered in the compositions of C133 and C331 model membranes: (A) POPG, (B) CL2, (C) DOMG, and (D) OPMG.

bilayer while the amine termini were oriented toward the membrane interphase. ${ }^{21,31}$ Based on this prior computations, two types of pores consisting of 16 aurein 1.2 molecules were built at the atomistic level with identical characteristics (Figure 2A).

One of the pore structures considered, $\mathbf{M}$, corresponds to a structure where the polar side of the peptides faces the pore interior (Figure 2B). In previous simulations, this type of pore self-assembled to render a structure with hydrophilic residues organized to face a cavity filled with water molecules and the hydrophobic residues facing the lipid tails. An alternative pore structure, FW, considered to evaluate the relevance of the orientation, corresponds to a pore where all the peptide molecules are translated in the $x y$ plane without involving any rotation, (which were oriented perpendicular to the bilayer and inserted into the two model membranes considered). The initial diameter of the pore structures was $3.0-3.5 \mathrm{~nm}$. The bilayer was composed of 512 lipids and the peptide/lipid (P/ L) ratio was $1: 32$, in line with literature and our previous studies. $^{21,30,31}$ In addition, peptides were placed in the aqueous solution at $3 \mathrm{~nm}$ far from the interphase with a C331 model membrane. This case is referred to as the $331 \mathrm{~S}$ system. Two bilayers without peptides were also considered and referred to as $\mathbf{N}$. A summary of the systems examined is presented in Table 2.

Table 2. Summary of the Systems Considered in This Study and Their Composition

\begin{tabular}{|c|c|c|}
\hline $\begin{array}{l}\text { system } \\
\text { name }\end{array}$ & system composition & $\begin{array}{l}\text { time } \\
(\mathrm{ns})\end{array}$ \\
\hline $331 N$ & $\begin{array}{l}384 \text { OPMG/96 POPG/16 CL2/18,052 TIP3/158 } \\
\mathrm{Na}^{+} / 30 \mathrm{Cl}^{-}\end{array}$ & 1000 \\
\hline $133 N$ & $\begin{array}{l}490 \mathrm{DOMG} / 16 \mathrm{POPG} / 3 \mathrm{CL} 2 / 17,985 \mathrm{TIP} 3 / 67 \mathrm{Na}^{+} / \\
45 \mathrm{Cl}^{-}\end{array}$ & 1000 \\
\hline $331 M$ & $\begin{array}{l}384 \text { OPMG/96 POPG/16 CL2/46,452 TIP3/214 } \\
\mathrm{Na}^{+} / 102 \mathrm{Cl}^{-} / 16 \text { peptides }\end{array}$ & 2300 \\
\hline $133 M$ & $\begin{array}{l}490 \mathrm{DOMG} / 16 \text { POPG/3 CL2/46,356 TIP3/107 } \\
\mathrm{Na}^{+} / 101 \mathrm{Cl}^{-} / 16 \text { peptides }\end{array}$ & 2300 \\
\hline $331 \mathrm{FW}$ & $\begin{array}{l}384 \text { OPMG/96 POPG/16 CL2/18,529 TIP3/161 } \\
\mathrm{Na}^{+} / 49 \mathrm{Cl}^{-} / 16 \text { peptides }\end{array}$ & 2300 \\
\hline $133 \mathrm{FW}$ & $\begin{array}{l}490 \mathrm{DOMG} / 16 \text { POPG/3 CL2/20,097 TIP } 3 / 58 \mathrm{Na}^{+} / \\
52 \mathrm{Cl}^{-} / 16 \text { peptides }\end{array}$ & 2300 \\
\hline $331 S$ & $\begin{array}{l}384 \text { OPMG/96 POPG/16 CL246,324 TIP3/214 } \\
\mathrm{Na}^{+} / 102 \mathrm{Cl}^{-} / 16 \text { peptides }\end{array}$ & 2400 \\
\hline \multicolumn{2}{|c|}{ total simulation time } & 13,600 \\
\hline
\end{tabular}

In what follows, the identification number of the strains and the initial pore conformation identifier will be used to

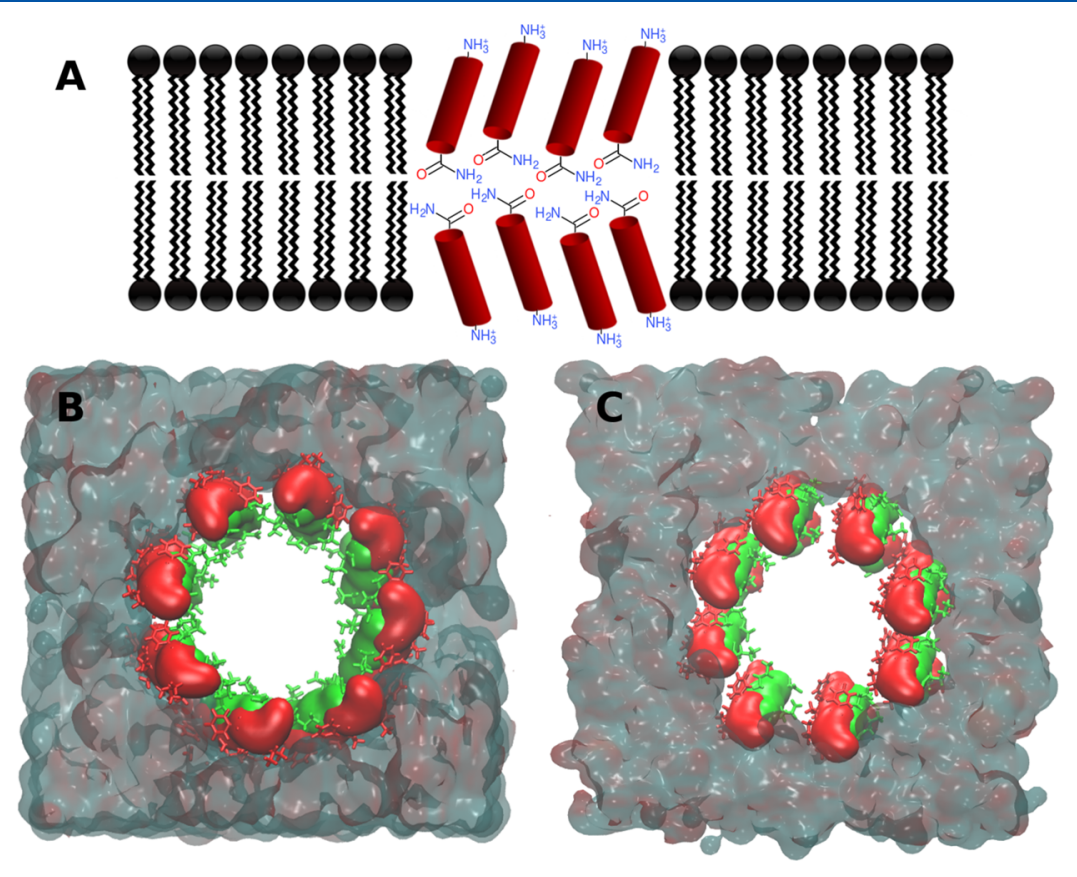

Figure 2. (A) Cartoon representation of the distribution of aurein 1.2 peptides in the lipid membrane. (B, C) Initial structural organization of the pore for the (B) $\mathbf{M}$ and (C) FW systems. Polar and charged peptide surfaces are depicted in green, and the nonpolar ones are in red. 

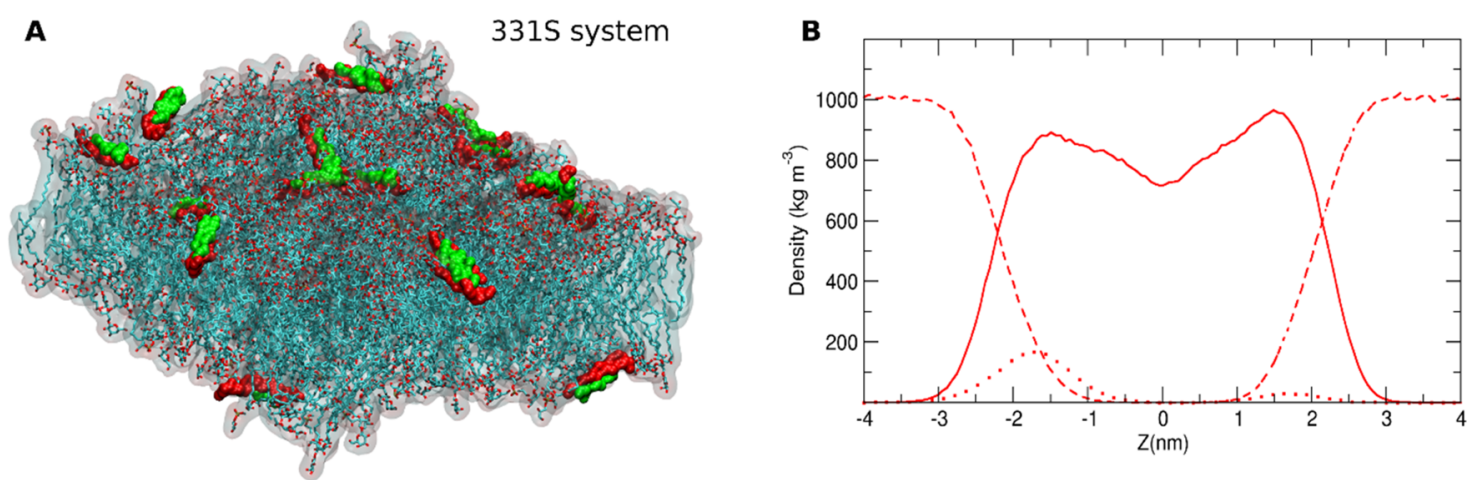

Figure 3. (A) Snapshot of the last frame $(2.3 \mu \mathrm{s})$ of the 331S system. Polar and charged peptide surfaces are depicted in green, and nonpolar surfaces are shown in red. Lipids are represented with sticks with a ghost surface representation. (B) Mass density profiles of water (dashed line), lipids (solid line), and peptides (dotted line) averaged over the last $500 \mathrm{~ns}$ of the simulations for the $331 \mathrm{~S}$ system.

denominate each system considered. For example, 331M refers to pore type $\mathbf{M}$ embedded in a $\mathbf{C} 331$ bilayer.

System Setup. The membrane builder module of CHARMM-GUI (http://www.charmm-gui.org/) was employed to generate the membranes and insert the pore structures into them using in-house scripts. The solvate CHARMM-GUI module was used to achieve a final concentration of $0.1 \mathrm{M} \mathrm{NaCl}$. MD simulations were performed with the GROMACS $2018.3^{52-56}$ software package. CHARMM $36^{57}$ parameters were used for ions, phospholipids, glycolipids, and amino acids, and the TIP3P model was used for water molecules.

The particle mesh Ewald (PME) ${ }^{58}$ method was used for the treatment of periodic electrostatic interactions with a cutoff distance of $1.2 \mathrm{~nm}$. The Lennard-Jones potential was smoothed over the cutoff range of $1.0-1.2 \mathrm{~nm}$ by using the force-based switching function. Only atoms in the Verlet pair list with a cutoff range reassigned every 20 steps were considered. The LINCS ${ }^{59}$ algorithm was used to constrain all bonds involving hydrogen atoms to allow the use of a $2 \mathrm{fs}$ time step. The protocol that is suggested by Lee et al. ${ }^{60}$ for nonbonded interactions with the CHARMM36 force field when used in the GROMACS suite was followed.

The systems were equilibrated in six stages with the peptides and lipids restrained using different force constants and relaxed progressively until the lipids are let free to move around a softly restrained protein backbone. The first $20 \mathrm{~ns}$ of the production run was discarded as part of the equilibration protocol. Production runs were performed in an $\mathrm{NP}_{X Y} \mathrm{P}_{Z} \mathrm{~T}$ ensemble. The temperature was maintained at $310 \mathrm{~K}$ with the Nosé-Hoover thermostat ${ }^{61,62}$ using a time constant of 1.0 ps. The pressure was maintained at 1 bar with the semi-isotropic Parrinello-Rahman barostat ${ }^{63}$ using a compressibility of $4.5 \times$ $10^{-5} \mathrm{bar}^{-1}$ and a time constant of $1.0 \mathrm{ps}$ in a rectangular simulation box. The simulation protocol employed is summarized in Table S2 of the Supporting Information.

Trajectory Analysis. Mass density profiles (MDPs) and density maps were calculated with the gmx density and gmx densmap tools from the GROMACS package. To quantify the content of peptide, water, and galactose inside the bilayer, a "hydrophobic region" between -1 and $+1 \mathrm{~nm}$ from the bilayer center was defined, and the MDP of these regions was calculated. The error bars were obtained by calculating the differential permeation at $0.1 \mathrm{~nm}$ outside and inside of the hydrophobic region limits. The total number of hydrogen bonds was calculated using the Hbonds VMD plugin, using a distance of $3 \AA$ and an angle of $30^{\circ}$ to define the hydrogen bond.

\section{RESULTS AND DISCUSSION}

The goal of this study was to understand from an atomistic point of view the differential susceptibility of the CIDCA 133 and CIDCA 331 strains to the AMP aurein 1.2. This phenomenon was observed experimentally in both living cells and bacterial-derived liposomes. ${ }^{41}$ The higher susceptibility of CIDCA 331 to aurein 1.2 was ascribed in part to its membrane composition. $^{42}$ Our hypothesis is that if a pore is formed, it will be more stable in a model membrane that emulates the CIDCA 331 strain rather than one that emulates the CIDCA 133 membrane. To test this hypothesis and to characterize the stability of preassembled aurein 1.2 pore structures embedded in two types of bilayers emulating the CIDCA 331 and CIDCA 133 probiotic strains, extensive atomistic MD simulations amounting to $13.6 \mu$ s were performed.

First, for the $331 \mathrm{~S}$ system where the peptides are initially placed in the aqueous solution in the vicinity of a model C331 bilayer, a $2.4 \mu$ s simulation was considered. As expected, pore formation did not realized at this time scale, and instead, the peptides were adsorbed at the water-lipid interphase (Figure $3 \mathrm{~A}$ ), as already reported in the literature studies for other peptides. $^{28,64}$ The peptide distribution at the water-lipid interphase is shown in Figure 3. According to mathematical studies of penetration, the size of the peptide ( $>9$ residues) and its physicochemical characteristics could lead to its insertion in mixture membranes. ${ }^{65}$ Their results indicate that peptides preconcentrate on the surface of the intact lipid mixture before jumping across it.

The time evolution of preassembled pore structures was followed. It has been found out that evolution of the specific properties studied is dependent on the composition of the membrane considered rather than the initial organization of the preassembled pore. The peptide pores were stabilized by hydrogen bonds established between the peptides and peptides with the water molecules inside the pores. Glycolipids played an important role by filling the pore fenestrations. Those peptide regions are suitable for binding polar galactose moieties, attracting them to the inner core of the membrane.

The evolution of the number of peptides in the inner core of the bilayer as a function of time for the $\mathbf{M}$ systems is shown in Figure 4. The number of peptides that constitute the pore remains constant during the simulation in the $331 \mathrm{M}$ case, which is at odds with the behavior of the peptides in the 133M 


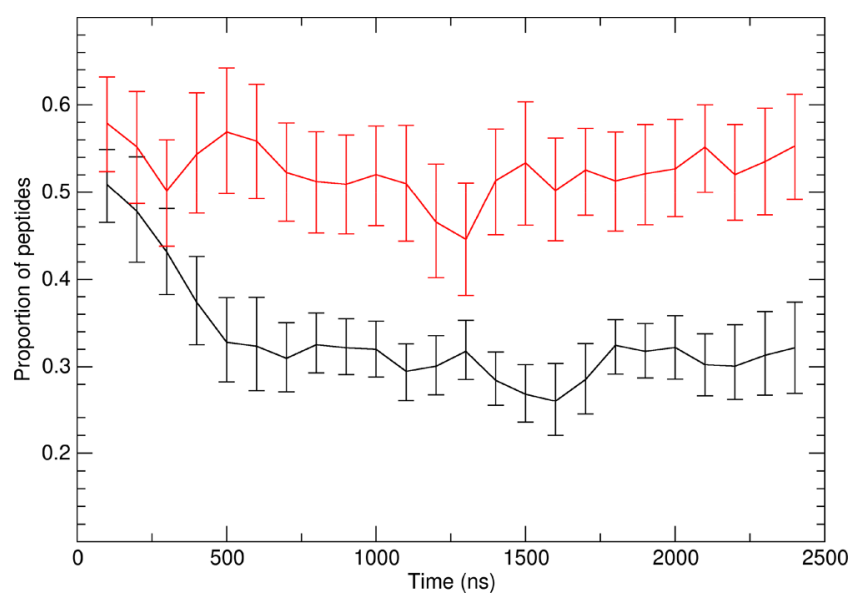

Figure 4. Proportion of peptides inside the hydrophobic core of the lipid membrane with respect to the total number of peptides in the simulation system as a function of simulation time. The red and black traces correspond to the $331 \mathrm{M}$ and $133 \mathrm{M}$ systems, respectively.

case where the proportion of peptides smoothly decreased during the first $600 \mathrm{~ns}$ of the simulations. In this way, the pore seems to be more stable in the C331 bilayer than in the C133 one. This could partially explain the susceptibility of C331 bilayers to the aurein 1.2 action. $^{41}$ Migration of individual peptides from the pore to the interphase was observed, especially in C133 bilayers. Once a peptide moves to the interphase, it stays there for the remaining simulation, as reported in similar studies. ${ }^{21,27,31}$ Furthermore, the peptides at the surface do not aggregate, but instead, they are adsorbed in the bilayer with the polar side chains oriented toward the water phase. Previously, similar aurein 1.2 behavior was reported from coarse grain MD simulation studies. ${ }^{21,31}$

The final representative structures of the pores in the simulations with bilayers $133 \mathrm{M}$ and $331 \mathrm{M}$ are illustrated in Figure 5. Despite the fact that the pore conformation was the same at the start of the simulation, the stability of the pore structure depends on the lipid composition; the $331 \mathrm{M}$ pore is stable in stark contrast with the $133 \mathrm{M}$ pore. In the later system, several aurein 1.2 peptides moved from the peptide assembly toward the interphase. Peptides in the 331FW and 133FW systems show generally the same behavior than those in the $331 \mathrm{M}$ and $133 \mathrm{M}$ systems, respectively. Nevertheless, the aurein 1.2 molecules rotated to align their polar faces toward the interior of the pore, resembling the $331 \mathrm{M}$ structure in the case of 331FW. This configuration enhanced the interaction with the water molecules inside the pore and stabilized its overall structure. In the $133 \mathrm{FW}$ case, some of the peptides migrate to the water-membrane interphase, like in the $133 \mathrm{M}$ case, but they induce a curvature in the bilayer.

Further information about the overall organization of the bilayers was obtained through the MDPs. The MDPs of the lipids and water in the $331 \mathrm{~N}$ and $133 \mathrm{~N}$ systems are shown in Figure 6A. Well-defined bilayers characterized by different

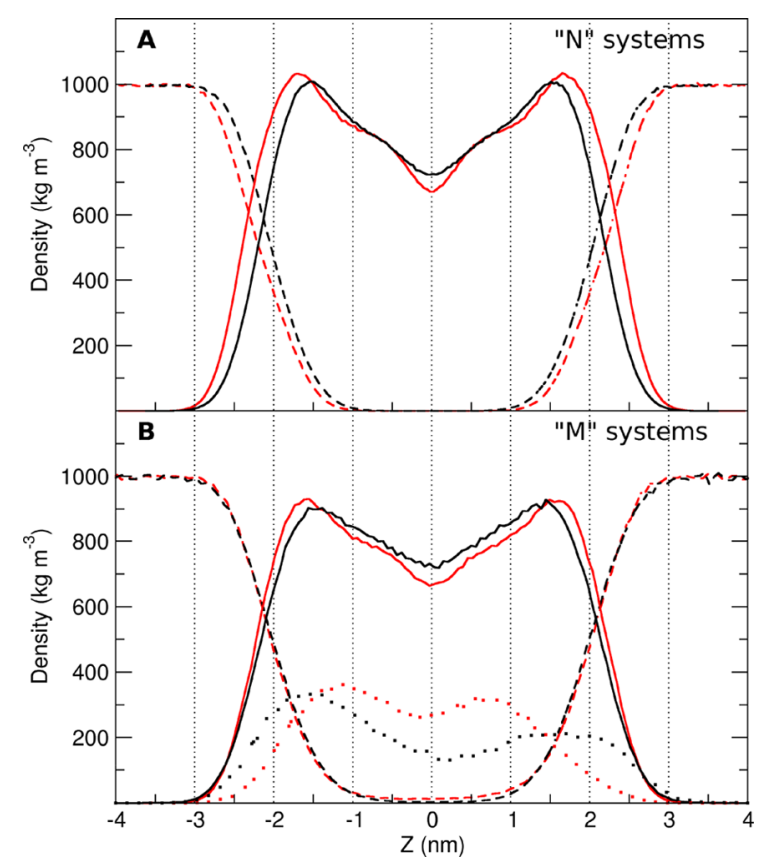

Figure 6. (A, B) Mass density profiles for water (dashed line), lipids (solid line), and peptides (dotted line) averaged over the last $500 \mathrm{~ns}$ of the simulations for (A) $\mathbf{N}$ and (B) $\mathbf{M}$ systems. Red and black traces represent C331 and C133 systems, respectively. Peptide densities are magnified five times for clarity.

degrees of thickness are found in the $\mathbf{N}$ systems. The thickness is defined as the distance between peaks in the total lipid density: $3.4 \pm 0.1$ and $3.0 \pm 0.1 \mathrm{~nm}$ for $331 \mathrm{~N}$ and $133 \mathrm{~N}$, respectively. These data together with the valley-to-peak ratio, deeper in the $331 \mathrm{~N}$ system, reveal a higher degree of organization in the $331 \mathrm{~N}$ bilayer in comparison to the $133 \mathrm{~N}$ system. This observation is expected if we consider the presence of glycolipids with a lower degree of tail unsaturation. Likewise, the average lipid area was found to be $57.8 \pm 0.6 \AA^{2}$ for the $331 \mathrm{~N}$ system compared to $64.9 \pm 0.7 \AA^{2}$ for $133 \mathrm{~N}$.

The MDPs of water, total lipids, and peptides for the $\mathbf{M}$ systems is shown in Figure 6B. In the presence of the pore, there is no significant difference between the thickness of the
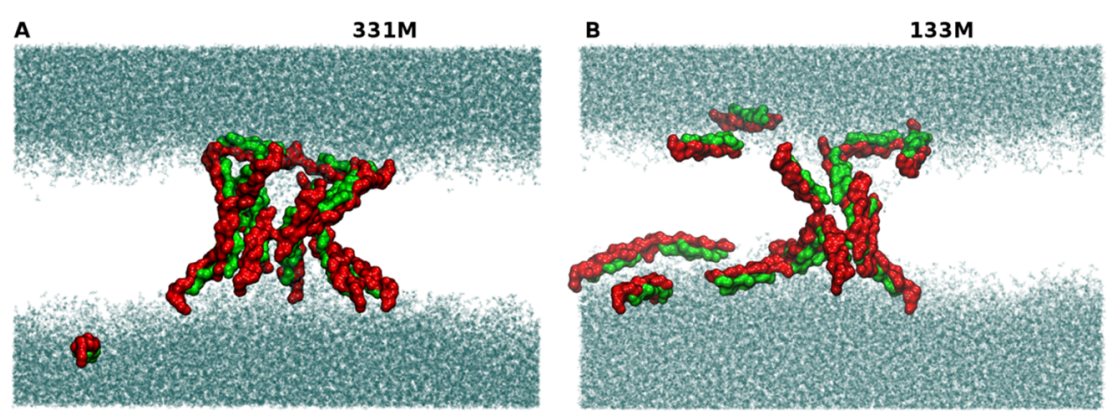

Figure 5. (A, B) Representative snapshots at $2.2 \mu$ s of the (A) $331 \mathrm{M}$ and (B) $133 \mathrm{M}$ systems. Polar and charged peptide surfaces are depicted in green, and nonpolar surfaces are shown in red. Water molecules are represented in cyan, and the lipid bilayer is not shown for clarity. 
$331 \mathrm{M}$ or $133 \mathrm{M}$ bilayers, $3.1 \pm 0.1$ and $3.0 \pm 0.1 \mathrm{~nm}$, respectively. This suggests different organization, in particular in the $\mathbf{C 3 3 1}$ bilayers. The peptide distribution confirms the difference in the pore assembly organization within the bilayer. Comparing the distribution of the peptides in each bilayer, inside the 331M bilayer, it is more uniform and denser compared to the $113 \mathrm{M}$ case where more peptides are found at the interphase. In contrast to what it is observed in wellorganized lipid membranes, the density of water is not negligible in the hydrophobic core regions as it was already described in other simulation studies of pore-like structures. ${ }^{66}$ The MDPs of all the systems considered are presented in the Supporting Information (Figures S2-S6).

The biological activity of these peptide structures relies on the transport of water through them. Indeed, water molecules were found inside the bilayer center when peptides were organized forming a pore inside the bilayer. Figure 7 shows the

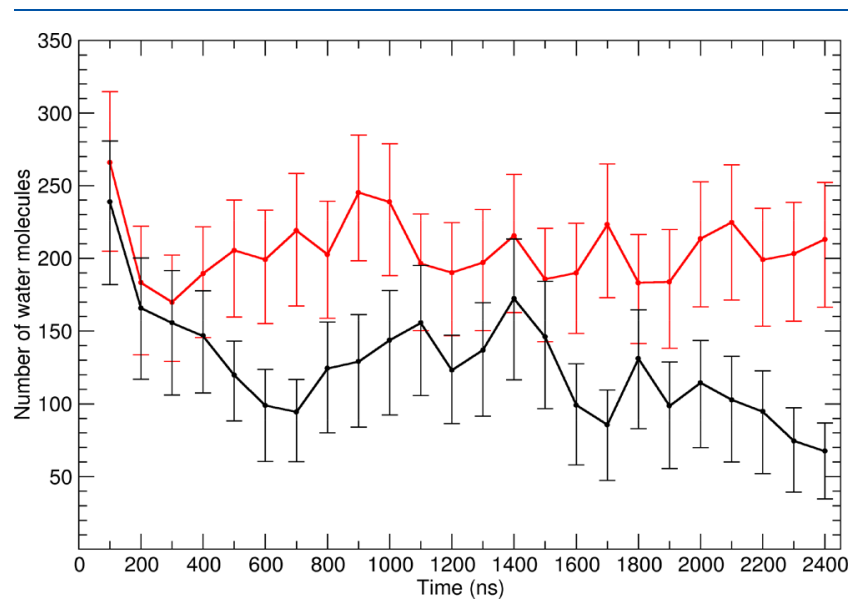

Figure 7. Number of water molecules inside the hydrophobic core of the lipid membrane as a function of simulation time. The red and black traces correspond to the $331 \mathrm{M}$ and $133 \mathrm{M}$ systems, respectively.

evolution of the number of water molecules in the inner core of the bilayer as a function of time for the $\mathbf{M}$ cases. The amount of water inside the pore remains stable for the $331 \mathrm{M}$ system, but it decreases for the 133M system. The pore structure in $331 \mathrm{M}$ remains stable, and water can easily permeate through it. In contrast, peptides migrated toward the interphase of the bilayer in the $133 \mathrm{M}$ case, and in parallel, the pore structure closes. This tendency was also observed in the 133FW and 331FW systems with some peculiarities. For instance, at the beginning of the simulations, some of the peptides oriented their polar regions toward the lipid hydrophobic tails, and as a result, the FW pores are less stable than the $\mathbf{M}$ ones. A slow spin of the peptides, which first increased the pore diameter with the concomitant water access permeation, was observed in 331FW (Figure S6A,B). This was followed by a pore relaxation resulting in a more organized and stable structure. The number of water molecules inside the pore decreases in the $133 \mathrm{FW}$ system as illustrated by the snapshots in Figure 8. As a result of the peptide organization, the membrane curvature changes, and the exact quantification of the number of water molecules is not viable.

In addition to the water flux, galactose headgroups are found inside the bilayer in the pore region. The evolution of the number of galactose molecules in the inner core of the bilayer as a function of time for the $\mathbf{M}$ cases is shown in Figure 9. The

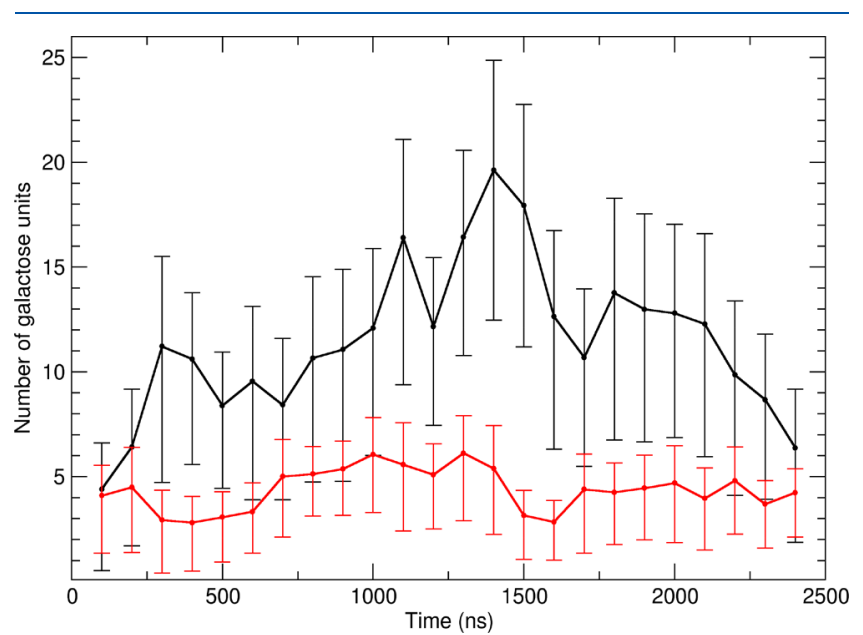

Figure 9. Number of galactose units inside the hydrophobic core of the lipid membrane as a function of simulation time. The red and black traces correspond to the $331 \mathrm{M}$ and $133 \mathrm{M}$ systems, respectively.

331M system, with a stable pore, shows, approximately, four galactose groups inside the hydrophobic region. In the case of $133 \mathrm{M}$, the number of sugars in this region increases, reaching a maximum value of $\sim 19$. This value decreases in parallel with the decrease of the size of the pore. Similar results were obtained for the FW systems, but the membrane ripples make its estimation difficult. The polar faces of the peptide structures are responsible for the presence of the galactose moieties in this region. Once there, they seem to promote the pore destabilization process by weakening peptide-peptide interactions. Considering that the two membranes have a high percentage of glycolipids, the difference in phospholipids may be the key in the differential stability.

To further characterize the contribution of galactose to AMP resistance, the number of hydrogen bonds (HB) between galactose and the peptides was analyzed as a function of simulation time. Galactose establishes $4 \pm 2$ and $6 \pm 2$ hydrogen bonds with the peptides in the $331 \mathrm{M}$ and 331FW systems, respectively (Figure 10A). The number remains constant during the simulation, which can be associated with the degree of pore stability in C331 membranes. In contrast,

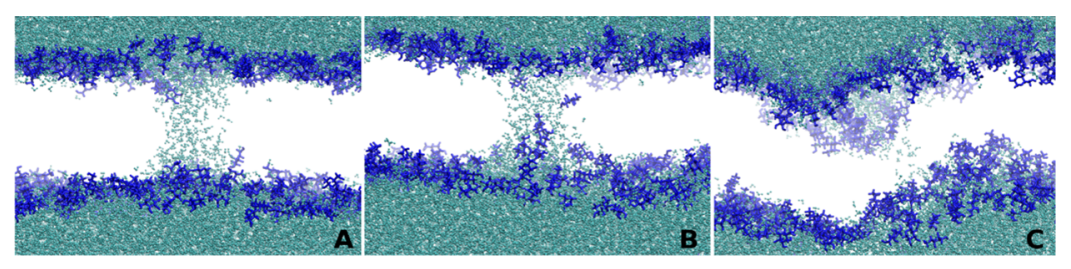

Figure 8. (A-C) Snapshots of the 133FW system (A) at the beginning of the simulation $(t=0 \mathrm{~ns}),(\mathrm{B})$ at $t=950 \mathrm{~ns}$, and (C) at the end of the simulation $(t=2200 \mathrm{~ns})$. Water is represented in cyan, and galactose is depicted in blue. 


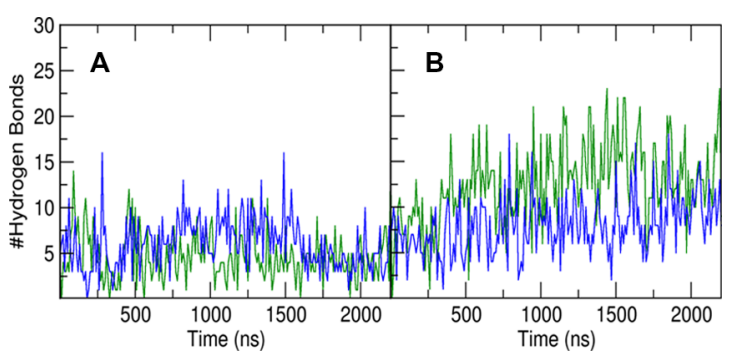

Figure 10. (A, B) Number of hydrogen bonds (HB) formed between the galactose glycolipid headgroups and the peptides in the (A) C331 and (B) C133 membranes. Green and blue traces correspond to the $\mathbf{M}$ and $\mathbf{F W}$ systems, respectively.

the number of hydrogen bonds when peptides are at the water-membrane interphase is higher; for instance, the average is $13 \pm 1$ in the $331 \mathrm{~S}$ system (Figure S9).

The HB time evolution for the 133M and 133FW systems is shown in Figure 10B. In both cases, the number of interactions increases with time despite starting at similar values. At the end of the simulation, at $2.3 \mu \mathrm{s}$, values in the range of $9-13$ are reached. These results are compatible with what it is expected from the pore evolution. In the C331 membrane, the pore remains assembled in a stable configuration, while in C133 membranes, the number of hydrogen bonds increases accounting for the migration of the peptide to the bilayer interphase, with the consequent pore narrowing. From the analysis of the interactions between the peptides and the lipid headgroups, it was found that aspartic and glutamic residues contribute the most to the interactions followed by serine and phenylalanine (Figure 11). Greater numbers of $\mathrm{HB}$ interactions are found in the $\mathbf{C 1 3 3}$ membranes as a result of the migration of the peptides to the interphase and the tendency of the glycolipid headgroups to penetrate the core of the membrane.

Lipid flip-flops were evaluated by tracking the position of the ether oxygen atom of the galactose group for glycolipids and the phosphorous atom of the phosphate group of the phospholipids. At least one glycolipid flip-flop event was identified in each simulation, and none was identified for phospholipids. The $z$ trajectories of two selected glycolipid ether oxygen atoms undergoing flip-flop in opposite directions in the 331FW system are shown in Figure 12A. Snapshots illustrating the flip-flop events in this simulation are shown in Figure 12B-D. The glycolipids involved in the flip-flop event are localized at the bilayer center, with the saccharide groups directly interacting with the peptides and the tails oriented toward the acyl lipid chains and parallel to the plane of the bilayer.

Phospholipid distribution in the $x y$ plane could play an important role on the differential stability of the pore in C311 membranes. The density maps of the glycolipids and phospholipids were calculated, and the backbone protein density map was used as a reference (Figure 13A). The glycolipid density map (Figure 13B) shows a wide homogeneous region (light green) that decreases in the vicinity of the pore (yellow and red). The phospholipid density map shows an uneven distribution with peptide accumulation at the edge of the pore (Figure 13C). In the C331 bilayer, the pore is surrounded by anionic phospholipids. Figures S10-S12 of the Supporting Information show the density maps of the other cases.
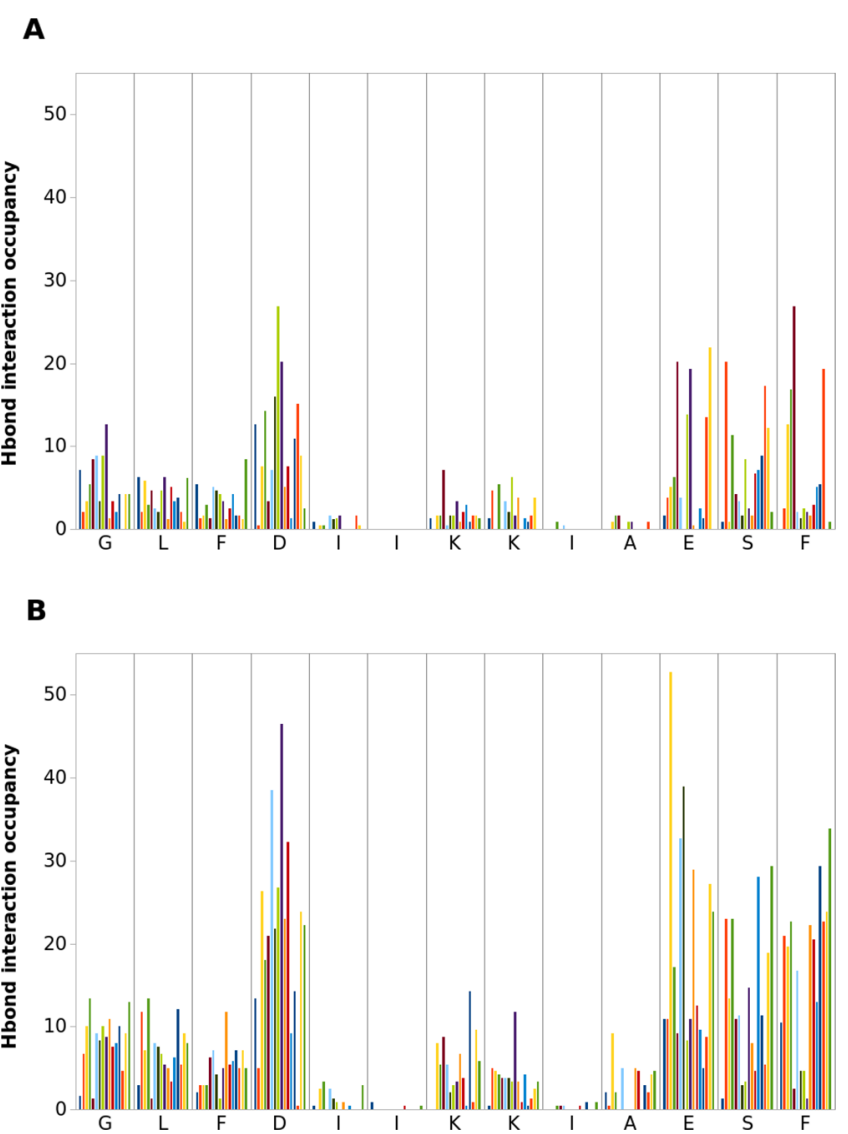

Figure 11. Occupancy percentage of peptide residue $\mathrm{HB}$ with lipid membrane groups. Each amino acid is expressed as one letter code, and there is one different color for the residue of each peptide. (A) C331 and (B) C133 systems.

The radial distribution function, $g(r)$, calculated between the phosphorous atoms of the phosphate groups of the phospholipids and the nitrogen atoms of either the side chain of lysine or the terminal group of glycine in the $331 \mathrm{M}$ and the 331FW systems is shown in Figure 14. A well-defined first peak at $\sim 4 \AA$, characteristic distance of salt bridge interactions, is observed. ${ }^{67}$ The interactions between peptides and charged phospholipids that could be partially responsible for maintaining the pore architecture were identified as salt bridges between these groups.

\section{CONCLUSIONS}

The aim of this work was to explore the mechanism of action of antimicrobial peptides in model membranes of two specific probiotic strains. We focused on the stability of the preassembled peptide pores in two model membranes. Considering our previous experimental findings and those by others, we expected greater stability in membranes that emulate the CIDCA 331 strain compared to those in the CIDCA 133 one. Our results are consistent with experimental reports on whole bacteria (CIDCA strains 331 and 133) and liposomes formed by lipids extracted from the aforementioned strains. ${ }^{41}$ In both cases, a greater susceptibility of strain 331 was observed with respect to the 133 strain against the action of aurein 1.2. In these experiments, the minimum inhibitory concentration (MIC) values for the CIDCA 133 strain were doubled those of the CIDCA 331 strain. The CIDCA 133 strain was able to grow at sub-MIC concentrations $(12 \mu \mathrm{M})$ of 
A
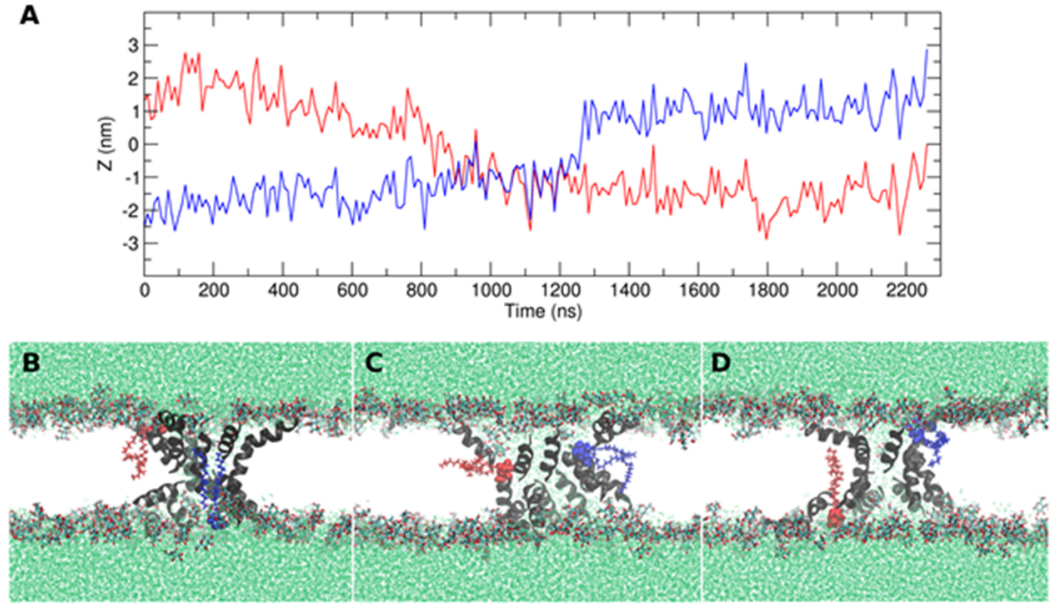

Figure 12. (A) Trajectories along the $z$ axis of ether oxygen atoms of the glycolipid headgroups of two selected molecules that participate in flipflop events in the 331FW system. (B-D) Snapshots of the system at (B) $600 \mathrm{~ns}$, (C) $1200 \mathrm{~ns}$, and (D) $2400 \mathrm{~ns}$. Water is shown in green, bulk lipid headgroups are shown in cyan/red, and peptides in cartoon representation are shown in black.
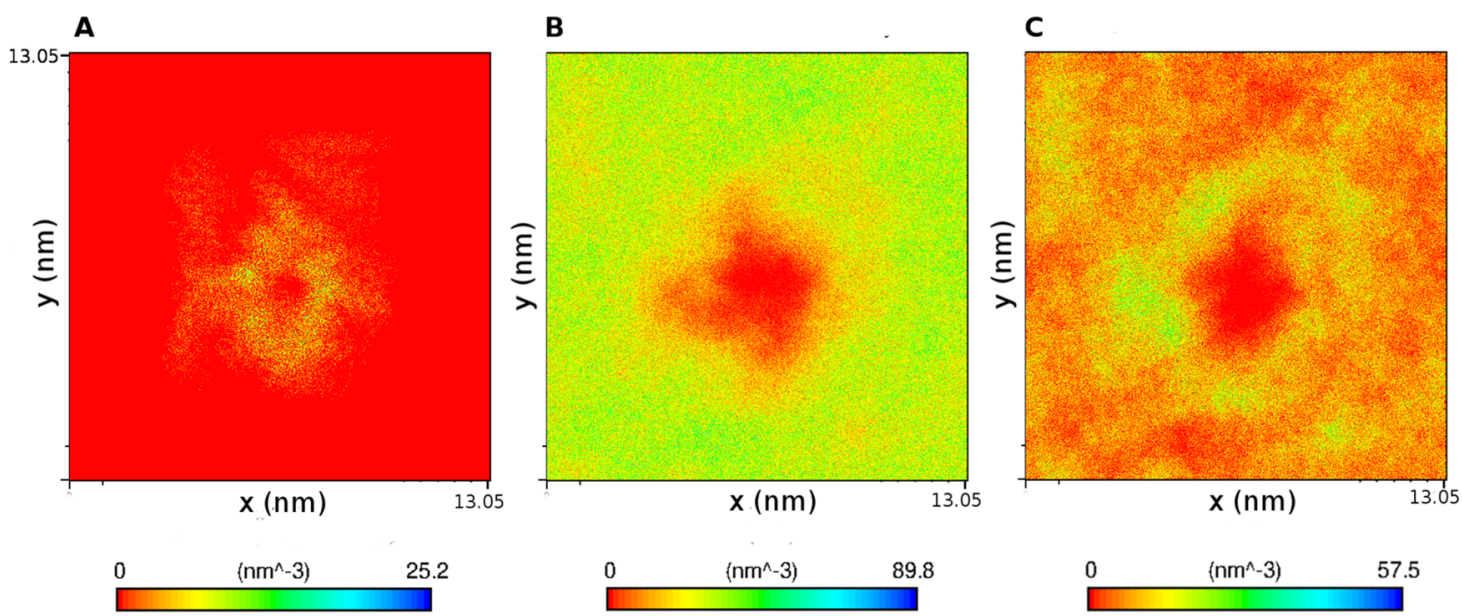

Figure 13. (A-C) Density maps for (A) peptide backbones, (B) glycolipids, and (C) phospholipids in the $x y$ plane of the $331 \mathrm{M}$ bilayer. Red represents the absence of molecules.

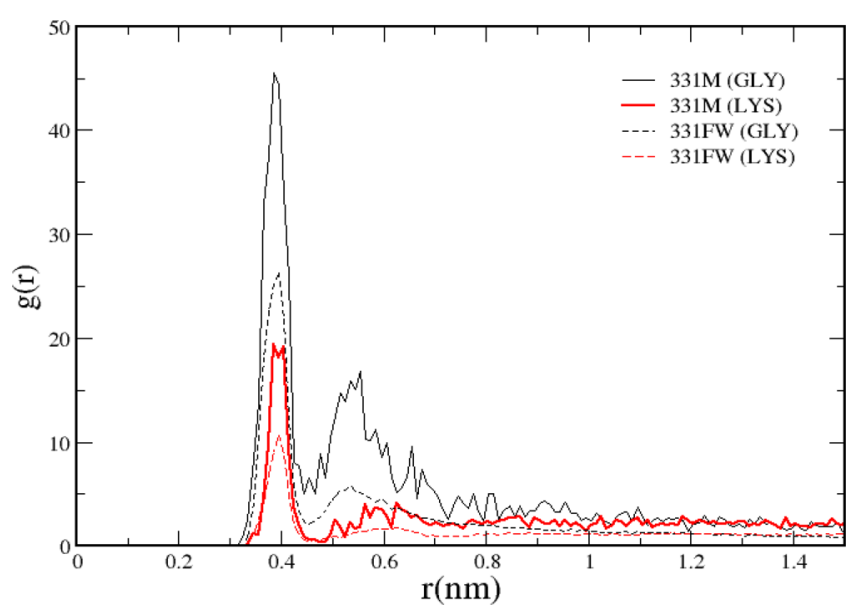

Figure 14. Radial distribution function between the phosphorous of the phospholipids and the amine nitrogen of the peptide terminal glycine (in black) and the side chain lysine nitrogen (red). Solid and dashed lines are used for $\mathbf{M}$ and $\mathbf{F W}$ systems, respectively.

aurein 1.2, while CIDCA 331 was significantly inhibited under the same conditions. Equally, the release of carboxyfluorescein of the large unilamellar vesicle at low concentrations is quantitatively higher for CIDCA 331 vesicles compared to CIDCA 133 ones, and the same was observed in the giant unilamellar vesicle with fluorescence microscopy. ${ }^{41}$ Likewise, our own computational CG simulation studies on prokaryotic model membranes ${ }^{21,31}$ provided insights into self-assembly and the formation of peptide pores. In this context, our hypothesis was formulated.

Our results show that the composition of the membrane is a crucial factor for the pore stability. The pores remain stable in the model membranes corresponding to CIDCA 331. However, some peptides migrated to the surface in the simulation of the CIDCA 133 system. Our results suggest that if a pore is formed by any mechanism, it will be more stable in the CIDCA 331 rather than in CIDCA 133 membranes. The mechanism by which the pore closes may be associated to the presence of a high number of glycolipids, present in both strains. The galactose unit has four hydroxyl groups, which are potential donor-acceptor of hydrogen atoms to establish specific interactions. However, the CIDCA 331 strain contains a higher percentage of negatively charged lipids (POPG and CL2). These anionic lipids surround the pore, shielding it from the glycolipid action. Further studies need to explore the 
transient aurein 1.2 pore lifetime as a function of the number of anionic phospholipids. A possible factor to be exploited to enhance or avoid the action of AMPs like aurein 1.2 is the presence of glycolipids.

This piece of work provides insight into the molecular hostprobiotic interactions. Several beneficial effects of probiotic microorganisms have been described in the literature studies. ${ }^{1,68-71}$ However, their mechanisms of action are diverse, heterogeneous, and strain specific and still not entirely understood. $^{72}$ Computer simulation studies, like the ones presented here, help to shed light on some of these aspects to maximize their applications in healthcare and food industries.

\section{ASSOCIATED CONTENT}

\section{SI Supporting Information}

The Supporting Information is available free of charge at https://pubs.acs.org/doi/10.1021/acs.jcim.0c00855.

More information about CIDCA 133/CIDCA 331 lipid composition; equilibration protocol, snapshots, and mass density profiles of all the systems; number of hydrogen bonds (331S system); and density maps (133M, 331FW, and 133FW systems) (PDF)

\section{AUTHOR INFORMATION}

\section{Corresponding Author}

Monica Pickholz - Departamento de Fisica Facultad de Ciencias Exactas y Naturales, Universidad de Buenos Aires, Ciudad Universitaria, Buenos Aires 1428, Argentina; IFIBA, CONICET-UBA, Ciudad Universitaria, Buenos Aires 1428, Argentina; 이이.org/0000-0002-0172-2748; Email: mpickholz@df.uba.ar

\section{Authors}

Galo E. Balatti - Departamento de Fisica Facultad de Ciencias Exactas y Naturales, Universidad de Buenos Aires, Ciudad Universitaria, Buenos Aires 1428, Argentina; IFIBA, CONICET-UBA, Ciudad Universitaria, Buenos Aires 1428, Argentina; (1) orcid.org/0000-0003-1900-1188

Carmen Domene - Department of Chemistry, University of Bath, Bath BA27AY, The United Kingdom; Department of Chemistry, University of Oxford, Oxford OX1 3TA, The United Kingdom; (1) orcid.org/0000-0001-7115-4232

M. Florencia Martini - Facultad de Farmacia y Bioquimica, Departamento de Farmacología, Universidad de Buenos Aires, C1113AAD Buenos Aires, Argentina; Instituto de Quimica y Metabolismo del Fármaco, Fac. de Farmacia y Bioquimica, Universidad de Buenos Aires, IQUIMEFA-CONICET, C1113AAD Buenos Aires, Argentina; 10 orcid.org/0000-00021949-9310

Complete contact information is available at:

https://pubs.acs.org/10.1021/acs.jcim.0c00855

\section{Author Contributions}

G.E.B. performed the simulations. All the authors were involved in the analysis and discussion of the data as well as in writing the article.

\section{Funding}

This work is funded by Argentina National Scientific and Technical Research Council (CONICET) grants: ANPCyT (PICT 2013-1205 and PICT 2014-3653).

\section{Notes}

The authors declare no competing financial interest.

\section{ACKNOWLEDGMENTS}

C.D. acknowledges the Partnership for Advanced Computing in Europe (PRACE) for an award to access computational resources in Daint at the Swiss National Supercomputing facility (https://www.cscs.ch/).

\section{ABBREVIATIONS}

CIDCA 133, Lactobacillus delbrueckii subsp. lactis; CIDCA 331, L. delbrueckii subsp. bulgaricus; CL2, 1',3'-bis[1-palmitoyl-2oleoyl-sn-glycero-3-phospho]-glycerol; CG, coarse grain; DMPC, 1,2-dimyristoyl-sn-glycero-3-phosphocholine; DMPG, 1,2-dimyristoyl-sn-glycero-3-phospho-(1'-rac-glycerol); DOMG, 1-palmitoyl-2-oleoyl-3-monogalactosyl-sn-glycerol; FW, ferris wheel; HB, hydrogen bonds; M, moon; MDP, mass density profile; $\mathrm{MD}$, molecular dynamics; $\mathrm{N}$, neat; OPMG, dioleoyl-3-monogalactosyl-sn-glycerol; PME, particle mesh Ewald; POPG, 1-palmitoyl-2-oleoyl-sn-glycero-3-phospho-(1'-rac-glycerol); S, surface

\section{REFERENCES}

(1) Hotel, A.; Cordoba, A. Health and Nutritional Properties of Probiotics in Food Including Powder Milk with Live Lactic Acid Bacteria. Prevention 2001, 1.

(2) Savage, D. C. Microbial Ecology of the Gastrointestinal Tract. Annu. Rev. Microbiol. 1977, 107.

(3) Kociubinski, G. L.; Pérez, P. F.; Añón, M. C.; De Antoni, G. L. A Method of Screening for Highly Inhibitory Lactic Acid Bacteria. J. Food Prot. 1996, 739.

(4) Kociubinski, G.; Pérez, P.; De Antoni, G. Screening of Bile Resistance and Bile Precipitation in Lactic Acid Bacteria and Bifidobacteria. J. Food Prot. 1999, 905.

(5) Hugo, A. A.; Kakisu, E.; De Antoni, G. L.; Pérez, P. F. Lactobacilli Antagonize Biological Effects of Enterohaemorrhagic Escherichia Coli in Vitro. Lett. Appl. Microbiol. 2008, 613.

(6) Hugo, A. A.; De Antoni, G. L.; Pérez, P. F. Lactobacillus Delbrueckii Subsp Lactis Strain CIDCA 133 Inhibits Nitrate Reductase Activity of Escherichia Coli. Int. J. Food Microbiol. 2006, 191.

(7) De Jesus, L. C. L.; Drumond, M. M.; de Carvalho, A.; Santos, S. S.; Martins, F. S.; Ferreira, Ê.; Fernandes, R. S.; de Barros, A. L. B.; do Carmo, F. L. R.; Perez, P. F.; Azevedo, V.; Mancha-Agresti, P. Protective Effect of Lactobacillus Delbrueckii Subsp. Lactis CIDCA 133 in a Model of 5 Fluorouracil-Induced Intestinal Mucositis. J. Funct. Foods 2019, 197.

(8) Hugo, A. A.; Rolny, I. S.; Romanin, D.; Pérez, P. F. Lactobacillus Delbrueckii Subsp. Lactis (Strain CIDCA 133) Stimulates Murine Macrophages Infected with Citrobacter Rodentium. World J. Microbiol. Biotechnol. 2017, 48.

(9) Wang, G.; Li, X.; Wang, Z. APD3: The Antimicrobial Peptide Database as a Tool for Research and Education. Nucleic Acids Res. 2016, 44, D1087-D1093.

(10) Reddy, K. V. R.; Yedery, R. D.; Aranha, C. Antimicrobial Peptides: Premises and Promises. Int. J. Antimicrob. Agents 2004, 536-547.

(11) Martin, E.; Ganz, T.; Lehrer, R. I. Defensins and Other Endogenous Peptide Antibiotics of Vertebrates. J. Leukocyte Biol. 1995, 58, 128-136.

(12) Jenssen, H.; Hamill, P.; Hancock, R. E. W. Peptide Antimicrobial Agents. Clin. Microbiol. Rev. 2006, 491-511.

(13) De La Fuente-Núñez, C.; Cardoso, M. H.; De Souza Cândido, E.; Franco, O. L.; Hancock, R. E. W. Synthetic Antibiofilm Peptides. Biochim. Biophys. Acta, Biomembr. 2016, 1061.

(14) Dathe, M.; Wieprecht, T. Structural Features of Helical Antimicrobial Peptides: Their Potential to Modulate Activity on Model Membranes and Biological Cells. Biochim. Biophys. Acta, Biomembr. 1999, 1462, 71-87. 
(15) Huang, Y.; Huang, J.; Chen, Y. Alpha-Helical Cationic Antimicrobial Peptides: Relationships of Structure and Function. Protein Cell 2010, 1, 143-152.

(16) Hancock, R. E. W.; Rozek, A. Role of Membranes in the Activities of Antimicrobial Cationic Peptides. FEMS Microbiol. Lett. 2002, 143-149.

(17) Haney, E. F.; Straus, S. K.; Hancock, R. E. W. Reassessing the Host Defense Peptide Landscape. Front. Chem. 2019, DOI: 10.3389/ fchem.2019.00043.

(18) Raheem, N.; Straus, S. K. Mechanisms of Action for Antimicrobial Peptides With Antibacterial and Antibiofilm Functions. Front. Microbiol. 2019, DOI: 10.3389/fmicb.2019.02866.

(19) Tossi, A.; Sandri, L.; Giangaspero, A. Amphipathic, AlphaHelical Antimicrobial Peptides. Biopolymers 2000, 55, 4-30.

(20) Rozek, T.; Wegener, K. L.; Bowie, J. H.; Olver, I. N.; Carver, J. A.; Wallace, J. C.; Tyler, M. J. The Antibiotic and Anticancer Active Aurein Peptides from the Australian Bell Frogs Litoria Aurea and Litoria Raniformis: The Solution Structure of Aurein 1.2. Eur. J. Biochem. 2000, 267, 5330-5341.

(21) Balatti, G.; Ambroggio, E.; Fidelio, G.; Martini, M.; Pickholz, M. Differential Interaction of Antimicrobial Peptides with Lipid Structures Studied by Coarse-Grained Molecular Dynamics Simulations. Molecules 2017, 22, 1775.

(22) Snider, C.; Jayasinghe, S.; Hristova, K.; White, S. H. MPEx: A Tool for Exploring Membrane Proteins. Protein Sci. 2009, 18, 26242628.

(23) Shahmiri, M.; Enciso, M.; Mechler, A. Controls and Constrains of the Membrane Disrupting Action of Aurein 1.2. Sci. Rep. 2015, 16378 .

(24) Fernandez, D. I.; Le Brun, A. P.; Whitwell, T. C.; Sani, M. A.; James, M.; Separovic, F. The Antimicrobial Peptide Aurein 1.2 Disrupts Model Membranes via the Carpet Mechanism. Phys. Chem. Chem. Phys. 2012, 15739.

(25) Gehman, J. D.; Luc, F.; Hall, K.; Lee, T. H.; Boland, M. P.; Pukala, T. L.; Bowie, J. H.; Aguilar, M. I.; Separovic, F. Effect of Antimicrobial Peptides from Australian Tree Frogs on Anionic Phospholipid Membranes. Biochemistry 2008, 47, 8557-8565.

(26) Rai, D. K.; Qian, S. Interaction of the Antimicrobial Peptide Aurein 1.2 and Charged Lipid Bilayer. Sci. Rep. 2017, 1.

(27) Poger, D.; Pöyry, S.; Mark, A. E. Could Cardiolipin Protect Membranes against the Action of Certain Antimicrobial Peptides? Aurein 1.2, a Case Study. ACS Omega 2018, 16453.

(28) Sharma, V. K.; Qian, S. Effect of an Antimicrobial Peptide on Lateral Segregation of Lipids: A Structure and Dynamics Study by Neutron Scattering. Langmuir 2019, 4152.

(29) McCubbin, G. A.; Praporski, S.; Piantavigna, S.; Knappe, D.; Hoffmann, R.; Bowie, J. H.; Separovic, F.; Martin, L. L. QCM-D Fingerprinting of Membrane-Active Peptides. Eur. Biophys. J. 2011, 437.

(30) Ambroggio, E. E.; Separovic, F.; Bowie, J. H.; Fidelio, G. D.; Bagatolli, L. A. Direct Visualization of Membrane Leakage Induced by the Antibiotic Peptides: Maculatin, Citropin, and Aurein. Biophys. J. 2005, 89, 1874-1881.

(31) Balatti, G. E.; Martini, M. F.; Pickholz, M. A Coarse-Grained Approach to Studying the Interactions of the Antimicrobial Peptides Aurein 1.2 and Maculatin 1.1 with POPG/POPE Lipid Mixtures. J. Mol. Model. 2018, 208.

(32) Yeaman, M. R.; Yount, N. Y. Mechanisms of Antimicrobial Peptide Action and Resistance. Pharmacol. Rev. 2003, 27.

(33) Von Deuster, C. I.; Knecht, V. Competing Interactions for Antimicrobial Selectivity Based on Charge Complementarity. Biochim. Biophys. Acta, Biomembr. 2011, 1808, 2867-2876.

(34) Fujii, G.; Eisenberg, D.; Selsted, M. E. Defensins Promote Fusion and Lysis of Negatively Charged Membranes. Protein Sci. 1993, 1301.

(35) Matsuzaki, K.; Sugishita, K. I.; Harada, M.; Fujii, N.; Miyajima, K. Interactions of an Antimicrobial Peptide, Magainin 2, with Outer and Inner Membranes of Gram-Negative Bacteria. Biochim. Biophys. Acta, Biomembr. 1997, 119.
(36) Matsuzaki, K.; Sugishita, K. I.; Ishibe, N.; Ueha, M.; Nakata, S.; Miyajima, K.; Epand, R. M. Relationship of Membrane Curvature to the Formation of Pores by Magainin 2. Biochemistry 1998, 37, 1185611863.

(37) Schmidt, N. W.; Wong, G. C. L. Antimicrobial Peptides and Induced Membrane Curvature: Geometry, Coordination Chemistry, and Molecular Engineering. Curr. Opin. Solid State Mater. Sci. 2013, 151.

(38) Bayer, A. S.; Prasad, R.; Chandra, J.; Koul, A.; Smriti, M.; Varma, A.; Skurray, R. A.; Firth, N.; Brown, M. H.; Koo, S. P.; Yeaman, M. R. In Vitro Resistance of Staphylococcus Aureus to Thrombin-Induced Platelet Microbicidal Protein Is Associated with Alterations in Cytoplasmic Membrane Fluidity. Infect. Immun. 2000, 3548.

(39) Schroeder, B. O.; Wu, Z.; Nuding, S.; Groscurth, S.; Marcinowski, M.; Beisner, J.; Buchner, J.; Schaller, M.; Stange, E. F.; Wehkamp, J. Reduction of disulphide bonds unmasks potent antimicrobial activity of human $\beta$-defensin 1. Nature 2011, 419.

(40) Seto, G. W. J.; Marwaha, S.; Kobewka, D. M.; Lewis, R. N. A. H.; Separovic, F.; McElhaney, R. N. Interactions of the Australian Tree Frog Antimicrobial Peptides Aurein 1.2, Citropin 1.1 and Maculatin 1.1 with Lipid Model Membranes: Differential Scanning Calorimetric and Fourier Transform Infrared Spectroscopic Studies. Biochim. Biophys. Acta, Biomembr. 2007, 1768, 2787-2800.

(41) Szymanowski, F.; Balatti, G. E.; Ambroggio, E.; Hugo, A. A.; Martini, M. F.; Fidelio, G. D.; Gómez-Zavaglia, A.; Pickholz, M.; Pérez, P. F. Differential Activity of Lytic $\alpha$-Helical Peptides on Lactobacilli and Lactobacilli-Derived Liposomes. Biochim. Biophys. Acta, Biomembr. 2019, 1069.

(42) Zavaglia, A. G.; Disalvo, E. A.; De Antoni, G. L. Fatty acid composition and freeze-thaw resistance in lactobacilli. J. Dairy Res. 2000, 67, 241-247.

(43) Hugo, A. A.; De Antoni, G. L.; Pérez, P. F. Lactobacillus Delbrueckii Subsp Lactis (Strain CIDCA 133) Resists the Antimicrobial Activity Triggered by Molecules Derived from Enterocyte-like Caco-2 Cells. Lett. Appl. Microbiol. 2010, 335.

(44) Hugo, A. A.; Tymczyszyn, E. E.; Gómez-Zavaglia, A.; Pérez, P. F. Effect of Human Defensins on Lactobacilli and Liposomes. J. Appl. Microbiol. 2012, 113, 1491-1497.

(45) Santo, K. P.; Berkowitz, M. L. Difference between Magainin-2 and Melittin Assemblies in Phosphatidylcholine Bilayers: Results from Coarse-Grained Simulations. J. Phys. Chem. B 2012, 116, 3021-3030.

(46) Cruz, V. L.; Ramos, J.; Melo, M. N.; Martinez-Salazar, J. Bacteriocin AS-48 Binding to Model Membranes and Pore Formation as Revealed by Coarse-Grained Simulations. Biochim. Biophys. Acta, Biomembr. 2013, 1828, 2524-2531.

(47) Catte, A.; Wilson, M. R.; Walker, M.; Oganesyan, V. S. Antimicrobial Action of the Cationic Peptide, Chrysophsin-3: A Coarse-Grained Molecular Dynamics Study. Soft Matter 2018, 2796.

(48) Sengupta, D.; Leontiadou, H.; Mark, A. E.; Marrink, S. J. Toroidal Pores Formed by Antimicrobial Peptides Show Significant Disorder. Biochim. Biophys. Acta, Biomembr. 2008, 2308.

(49) Balatti, G. E.; Martini, M. F.; Pickholz, M. Insights on the Antimicrobial Peptides Transient Pores Life as Function of the Ratio Glicolipid/Phospholipids on Model Membranes: A Coarse-Grained Molecular Dynamics Simulations Approach. Unpublished Manuscript.

(50) Touw, W. G.; Baakman, C.; Black, J.; te Beek, T. A. H.; Krieger, E.; Joosten, R. P.; Vriend, G. A Series of PDB-Related Databanks for Everyday Needs. Nucleic Acids Res. 2010, 43, D1.

(51) Marcotte, I.; Wegener, K. L.; Lam, Y. H.; Chia, B. C. S.; De Planque, M. R. R.; Bowie, J. H.; Auger, M.; Separovic, F. Interaction of Antimicrobial Peptides from Australian Amphibians with Lipid Membranes. Chem. Phys. Lipids 2003, 122, 107-120.

(52) Abraham, M. J.; Murtola, T.; Schulz, R.; Páll, S.; Smith, J. C.; Hess, B.; Lindahl, E. Gromacs: High Performance Molecular Simulations through Multi-Level Parallelism from Laptops to Supercomputers. SoftwareX 2015, 1-2, 19.

(53) Pronk, S.; Páll, S.; Schulz, R.; Larsson, P.; Bjelkmar, P.; Apostolov, R.; Shirts, M. R.; Smith, J. C.; Kasson, P. M.; Van Der 
Spoel, D.; Hess, B.; Lindahl, E. GROMACS 4.5: A High-Throughput and Highly Parallel Open Source Molecular Simulation Toolkit. Bioinformatics 2013, 29, 845-854.

(54) Van Der Spoel, D.; Lindahl, E.; Hess, B.; Groenhof, G.; Mark, A. E.; Berendsen, H. J. C. GROMACS: Fast, Flexible, and Free. J. Comput. Chem. 2005, 1701.

(55) Berendsen, H. J. C.; van der Spoel, D.; van Druded, R. GROMACS: A Message-Passing Parallel Molecular Dynamics Implementation. J. Comput. Chem. 1995, 91, 43-56.

(56) Hess, B.; Kutzner, C.; Van Der Spoel, D.; Lindahl, E. GRGMACS 4: Algorithms for Highly Efficient, Load-Balanced, and Scalable Molecular Simulation. J. Chem. Theory Comput. 2008, 4, 435-447.

(57) Huang, J.; Mackerell, A. D., Jr. CHARMM36 All-Atom Additive Protein Force Field: Validation Based on Comparison to NMR Data. J. Comput. Chem. 2013, 34, 2135.

(58) Petersen, H. G. Accuracy and Efficiency of the Particle Mesh Ewald Method. J. Chem. Phys. 1995, 103, 3668-3679.

(59) Hess, B.; Bekker, H.; Berendsen, H. J. C.; Fraaije, J. G. E. M. LINCS: A Linear Constraint Solver for Molecular Simulations. J. Comput. Chem. 1997, 18, 1463.

(60) Lee, J.; Cheng, X.; Swails, J. M.; Yeom, M. S.; Eastman, P. K.; Lemkul, J. A.; Wei, S.; Buckner, J.; Jeong, J. C.; Qi, Y.; Jo, S.; Pande, V. S.; Case, D. A.; Brooks, C. L., III; MacKerell, A. D., Jr.; Klauda, J. B.; Im, W. CHARMM-GUI Input Generator for NAMD, GROMACS, AMBER, OpenMM, and CHARMM/OpenMM Simulations Using the CHARMM36 Additive Force Field. J. Chem. Theory Comput. 2016, 12, 405.

(61) Nosé, S. A Unified Formulation of the Constant Temperature Molecular Dynamics Methods. J. Chem. Phys. 1984, 81, 511.

(62) Hoover, W. G. Canonical Dynamics: Equilibrium Phase-Space Distributions. Phys. Rev. A 1985, 31, 1695-1697.

(63) Parrinello, M.; Rahman, A. Polymorphic Transitions in Single Crystals: A New Molecular Dynamics Method. J. Appl. Phys. 1981, 52, 7182.

(64) Sun, D.; Forsman, J.; Woodward, C. E. Amphipathic Membrane-Active Peptides Recognize and Stabilize Ruptured Membrane Pores: Exploring Cause and Effect with Coarse-Grained Simulations. Langmuir 2015, 31, 752.

(65) Ramírez, P. G.; Del Pópolo, M. G.; Vila, J. A.; Szleifer, I.; Longo, G. S. Adsorption and Insertion of Polyarginine Peptides into Membrane Pores: The Trade-off between Electrostatics, Acid-Base Chemistry and Pore Formation Energy. J. Colloid Interface Sci. 2019, $552,701$.

(66) Albano, J. M. R.; Mussini, N.; Toriano, R.; Facelli, J. C.; Ferraro, M. B.; Pickholz, M. Calcium Interactions with Cx26 Hemmichannel: Spatial Association between MD Simulations Biding Sites and Variant Pathogenicity. Comput. Biol. Chem. 2018, 77, 331.

(67) Wood, I.; Martini, M. F.; Pickholz, M. Similarities and Differences of Serotonin and Its Precursors in Their Interactions with Model Membranes Studied by Molecular Dynamics Simulation. J. Mol. Struct. 2013, 1045, 124.

(68) Begum, P.; Madhavi, G.; Rajagopal, S.; Viswanath, B.; Razak, M.; Venkataratnamma, V. Probiotics as Functional Foods: Potential Effects on Human Health and Its Impact on Neurological Diseases. Int. J. Nutr., Pharmacol., Neurol. Dis. 2017, 7, 23.

(69) Parvez, S.; Malik, K. A.; Ah Kang, S.; Kim, H. Y. Probiotics and Their Fermented Food Products Are Beneficial for Health. J. Appl. Microbiol. 2006, 100, 1171.

(70) Nagpal, R.; Kumar, A.; Kumar, M.; Behare, P. V.; Jain, S.; Yadav, H. Probiotics, Their Health Benefits and Applications for Developing Healthier Foods: A Review. FEMS Microbiol. Lett. 2012, 1.

(71) Hill, C.; Guarner, F.; Reid, G.; Gibson, G. R.; Merenstein, D. J.; Pot, B.; Morelli, L.; Canani, R. B.; Flint, H. J.; Salminen, S.; Calder, P. C.; Sanders, M. E. Expert Consensus Document: The International Scientific Association for Probiotics and Prebiotics Consensus Statement on the Scope and Appropriate Use of the Term Probiotic. Nat. Rev. Gastroenterol. Hepatol. 2014, 11, 506.
(72) Plaza-Diaz, J.; Ruiz-Ojeda, F. J.; Gil-Campos, M.; Gil, A. Mechanisms of Action of Probiotics. In Advances in Nutrition; 2019, DOI: $10.1093 /$ advances/nmy063. 